\title{
Formation of Three-carbon Sugar Fragment at an Early Stage of the Browning Reaction of Sugar with Amines or Amino Acids
}

\author{
Tateki Hayashi, Sadako MASE and Mitsuo NAmIKI \\ Department of Food Science \& Technology, Faculty of Agriculture, \\ Nagoya University, Chikusa-ku, Nagoya 464, Japan
}

Received December 23, 1985

\begin{abstract}
Formation of a product easily converted to methylglyoxal on TLC with silica gel was observed in an early stage of the reaction mixture of sugar with an alkylamine or amino acid. NMR spectra of the ether extract of reaction mixtures indicated that methylglyoxal dialkylimine was produced mainly at an early stage of the reaction of glucose with alkylamine, and was assumed to change to methylglyoxal on the TLC. The $\mathrm{C}_{3}$ imine production in the $t$-butylamine system was apparently little and slow compared to that in the normal alkylamine system. A large, rapid production of $\mathrm{C}_{3}$ imine was also observed in the system of the Amadori product and $n$-butylamine. These results suggested that the $\mathrm{C}_{3}$ formation in the system with normal alkylamine may occur mainly via a newly proposed mechanism, though the $t$-butylamine system may possibly produce it according to the scheme proposed by Hodge.
\end{abstract}

As previously reported, ${ }^{1,2)}$ we have found that a novel free radical product was formed at an early stage in the browning reactions of sugar-amino compound systems, and ESR analyses led to the assignment of an $N, N^{\prime}$ disubstituted pyrazine cation radical structure. This structure reasonably led us to the assumption of the formation of an $\mathrm{C}_{2}$ fragmentation product of the material sugar as a precursor of the radical product, and this was demonstrated by isolation and identification of glyoxal dialkylimine. ${ }^{3)}$ Based on various other experimental evidence ${ }^{4,5)}$ concerning sugar fragmentation and free radical formation, we have proposed a new pathway for browning that involves cleavage of the sugar moiety of the Schiff base at an initial stage of the aminocarbonyl reaction before the Amadori rearrangement, followed by formation of the free radical and $\mathrm{C}_{2}$ products. Besides the formation of $\mathrm{C}_{2}$ product, we have also recognized the presence of a $\mathrm{C}_{3}$ compound as another fragmentation product in an early stage of the sugar-amine reaction.

In the browning reaction mechanism proposed by Hodge, ${ }^{6)}$ no consideration has been given to sugar fragmentation producing reactive intermediates of browning, especially at an early stage, except for a postulation of a $\mathrm{C}_{3}$ carbonyl compound as one of the active products formed from 1-deoxyhexosones in the middle stage of the reaction. This study deals with the identification of the $\mathrm{C}_{3}$ fragmentation product of sugar at an early stage of the amino-carbonyl reaction, and a proposal of its formation pathway.

\section{RESULTS AND DISCUSSION}

When mixtures of various sugar-alkylamine systems at an early stage of the reaction were developed on silica gel TLC plates and sprayed with 2,4-DNP solution, two particular orange spots appeared instantaneously (Fig. 1). The lower one observed in every system has previously been identified as glyoxal, and was shown to be an artifact formed from glyoxal dialkylimine by hydrolysis with silica gel. ${ }^{3)}$ An additional spot with a higher $R f$ value which appeared in the systems with alkyl amines other than $t$-butylamine has remained to be identified. 


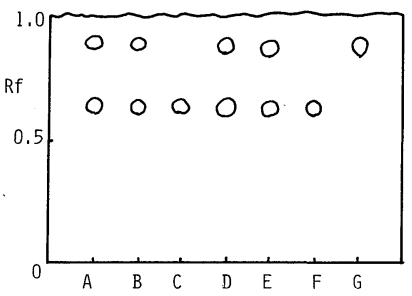

Fig. 1. TLC of Reaction Mixtures of Sugars with Amines.

Mixtures of the materials in distilled water (1 $\mathrm{M}$ each) were heated for $10 \mathrm{~min}$ in a boiling water bath, developed on silica gel TLC plates, and sprayed with 2,4-DNP reagent. A, glucose + ethylamine; B, glucose + cyclohexylamine; C, glucose $+t$-butylamine; $\mathrm{D}, \quad$ glucose $+n$-butylamine; $\mathrm{E}$, xylose $+n$-butylamine; F, glyoxal; $\mathrm{G}$, methylglyoxal.

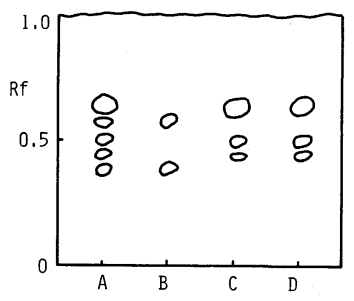

FIG. 2. TLC of the Phenylhydrazones of the Reaction Products.

TLC: silica gel, benzene- $n$-hexane-ethyl acetate $(5: 2$ : 1). A, ether extract of the reaction mixture of glucose and n-butylamine; B, authentic glyoxal phenylhydrazone; $\mathrm{C}$, isolated substance; $\mathrm{D}$, authentic methylglyoxal phenylhydrazone.

An ether extract of the reaction mixture of glucose and $n$-butylamine was treated with phenylhydrazine reagent. The obtained phenylhydrazones gave 5 spots on TLC (Fig. 2-A), and two of them have been identified as conformational isomers of glyoxal bisphenylhydrazone (B). The topmost spot on TLC was a new main one and was isolated by preparative TLC to give a yellow crystalline product. The product gave three spots on the same TLC corresponding to methylglyoxal phenylhydrazones even after further purification by preparative HPLC (C), and they agreed well in their $R f$ values to those observed with authentic methylglyoxal phenylhydrazone (D in Fig. 2), indicating the presence of its conformational isomers. Confirmatory evidence was that NMR data of the isolated phenyl-
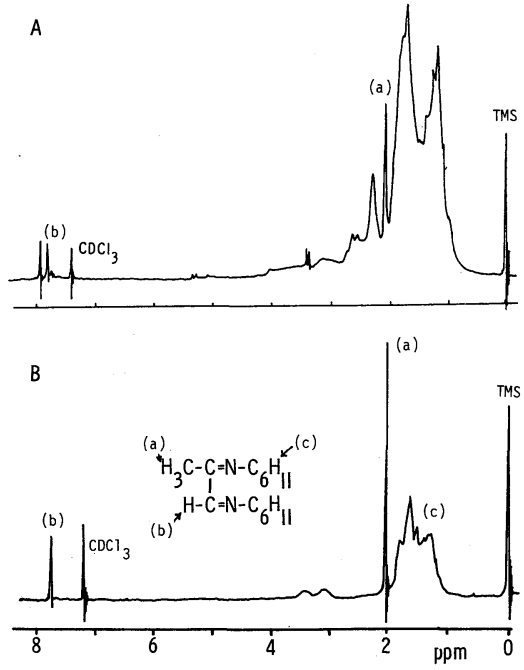

FIG. 3. NMR Spectra of the Ether Extract of the Reaction Mixture of Glucose and Cyclohexylamine (A), and Authentic Methylglyoxal Dicyclohexylimine (B).

hydrazone was identical to that of the authentic one.

Methylglyoxal was also expected to be present as its dialkylimine form in the reaction mixture, as was the case with glyoxal. ${ }^{3)}$ Many trials to isolate it from the reaction mixture using TLC and HPLC were unsuccessful, perhaps due to its instability. However, the presence of methylglyoxal dialkylimine in the early stage of the reaction could be demonstrated by comparison of its spectral features with those of the authentic one as follows.

Methylglyoxal dicyclohexylimine was synthesized by the reaction of methylglyoxal with cyclohexylamine as unstable crystals. The MS spectrum gave $\mathrm{M}^{+}$of 234 corresponding to the Schiff base-type condensation product of 2 molecules of cyclohexylamine with 1 molecule of methylglyoxal. A large peak at $m / z 191$ can be explained by the fragment

$$
\mathrm{CH}_{2}=\mathrm{CH}-\mathrm{CH}=\mathrm{N}=\mathrm{C}\left(\mathrm{CH}_{3}\right)-\mathrm{CH}=\mathrm{N}-\mathrm{C}_{6} \mathrm{H}_{11}
$$

resulting from ring fission of the cyclohexylamine derivatives. ${ }^{7)}$ The NMR spectrum (Fig. 3B) showed proton signals for two cyclohexyl groups $(1.3 \sim 1.8,3.1$ and $3.4 \mathrm{ppm})$, a signal for one methyl group (2.08 ppm), and one for a proton of the imine group $(7.8 \mathrm{ppm})$, indicat- 
ing that the synthesized one is methylglyoxal dicyclohexylimine.

The NMR spectrum of the ether extract of the reaction mixture of cyclohexylamine with glucose (Fig. 3A) showed signals identical to those of synthetic methylglyoxal dicyclohexylimine in addition to those due to glyoxal dicyclohexylimine (7.9 ppm, imine protons), though it also contained the signals due to cyclohexylamine $(1 \sim 2.7 \mathrm{ppm})$. The MS spectrum of the ether extract also gave $\mathrm{M}^{+}$of 234 corresponding to methylglyoxal dicyclohexylimine. When the authentic methylglyoxal dicyclohexylimine was developed on a silica gel plate as was done for the reaction mixture, only one spot was detected by $2,4-\mathrm{DNP}$ at the same $R f$ value as that of methylglyoxal. Moreover, methylglyoxal dicyclohexylimine treated with phenylhydrazine reagent gave three spots identical to those found in C and D in Fig. 2. These results indicated that methylglyoxal dicyclohexylimine is one of the main products in an early stage of the reaction of glucose with cyclohexylamine, and that most of the methylglyoxal detected on TLC does not exist as such in the reaction mixture.

Formation of methylglyoxal di- $n$-butylimine by the reaction of glucose with $n$-butylamine was also observed by NMR. NMR of $\mathrm{CDCl}_{3}$ extract from the reaction mixture gave signals corresponding to those of the ether extract of the reaction mixture of methylglyoxal with $n$ butylamine. All the signals due to methyl and imine groups were identical to those of the glucose-cyclohexylamine system.

It was shown by the experiment using GC of the reaction mixture of glucose with $\beta$-alanine, treated with $o$-phenylenediamine, that methylglyoxal and glyoxal were also detected as methylquinoxaline and quinoxaline, respectively (Fig. 4). There might be exchange reactions of amino compounds attached to carbonyl groups between the amino acid and $o$ phenylenediamine. These results indicated that methylglyoxal diimine may be produced by the reaction of sugar with amino compounds. Participation of alkaline degradation of sugar alone in $\mathrm{C}_{3}$ formation is considered to be
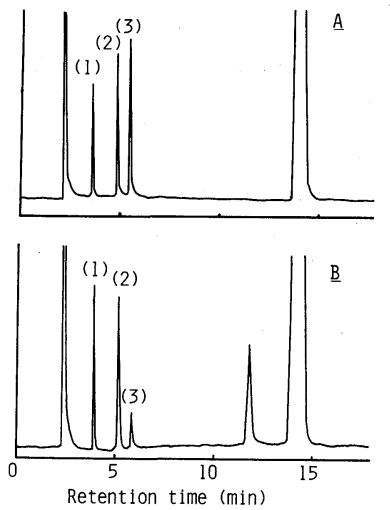

FIG. 4. Capillary GC of Products from the Reaction of Glucose with $\beta$-Alanine.

A, mixture of glyoxal (2) and methylglyoxal (3); B, reaction mixture in water $(2 \mathrm{M}$ each, initial $\mathrm{pH} 9.29)$ heated for $20 \mathrm{~min}$ in a boiling water bath. Each sample contains naphthalene (1) as an internal standard.

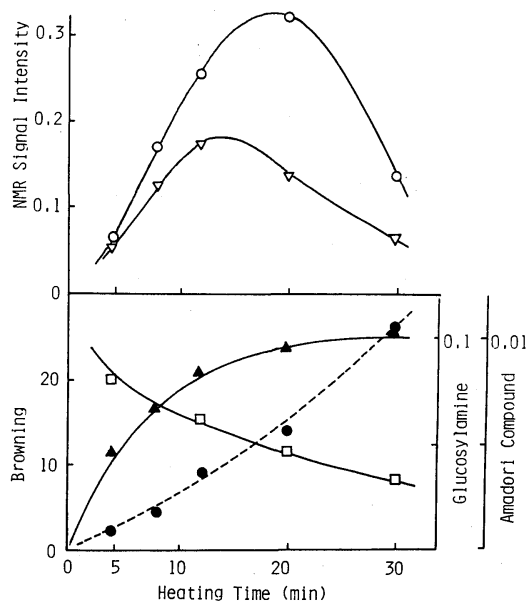

FIG. 5. Formation of $\mathrm{C}_{2}$ Imine, $\mathrm{C}_{3}$ Imine, and Other Intermediate Products by the Reaction of Glucose with $n$ Butylamine (1 $\mathrm{M}$ each) heated at $80^{\circ} \mathrm{C}$.

$-\nabla-, \mathrm{C}_{2}$ imine; - $\mathrm{-}-\mathrm{C}_{3}$ imine; $-\square-$, glucosylamine; $-\mathbf{\Delta}-$, Amadori compound: --- ---, browning.

negligibly small because methylglyoxal was not detected by GC in the alkaline solution of glucose $(2 \mathrm{M}, \mathrm{pH} 9.3)$ heated at $95^{\circ} \mathrm{C}$ for 10 min.

Quantitative studies of the course of formation of the $\mathrm{C}_{3}$ imine in the glucose- $n$-butylamine system indicated that $\mathrm{C}_{3}$ compound production occurred at a very early stage, increased with heating time giving a maximum 
at about $20 \mathrm{~min}$ and then gradually decreased (Fig. 5). The course of the production of $\mathrm{C}_{3}$ imine was closely followed by that of $\mathrm{C}_{2}$ imine, and well correlated to the decrease in the amount of glucosylamine and the increase in the formation of Amadori product. Reaction of Amadori product with $n$-butylamine rapidly produced the $\mathrm{C}_{3}$ compound in a manner similar to that of the glucose- $n$-butylamine system (Fig. 6). These results indicate the possibility that the Amadori product participates in the $\mathrm{C}_{3}$ formation.

As mentioned in Fig. 1, a spot corresponding to methylglyoxal was hardly detected on TLC in the glucose- $t$-butylamine system. Production of the $\mathrm{C}_{2}$ and $\mathrm{C}_{3}$ compound and the rate of browning in this system were compared with those of the $n$-butylamine system during the course of the reaction. As shown in Fig. 7, the formation of $\mathrm{C}_{2}$ imine in the $t$-butylamine system was very rapid and

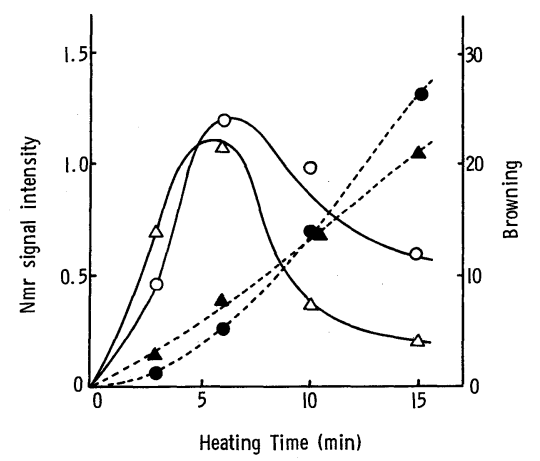

FIG. 6. Formation of $\mathrm{C}_{3}$ Imine by the Reaction of $n$ Butylamine with Amadori Compound or Glucose.

Each mixture in water was adjusted to $\mathrm{pH} 10.8$. Glucose $(0.5 \mathrm{M})+n$-butylamine $(1 \mathrm{M})$ : $-\mathrm{O}-, \mathrm{C}_{3}$ imine; --- --browning. Amadori compd. $(0.5 \mathrm{M})+n$-butylamine $(0.5 \mathrm{M})$ : $-\triangle-, C_{3}$ imine; --- $\mathbf{\Delta - - -}$, browning. similar in its course to that in the system of $n$ butylamine though the yield was relatively low. However, in the case of $\mathrm{C}_{3}$ imine formation, it was significantly suppressed and delayed in the $t$-butylamine system compared to that in the $n$-butylamine system. This apparent difference is probably due to the effect of the bulkier structure of $t$-butylamine on the reactivity in amino-carbonyl reaction with sugar. If we suppose a pathway of sugar fragmentation to yield $\mathrm{C}_{3}$ imine by a reversealdol condensation type reaction of the further aminated Amadori product (Fig. 8), there is a significant difference in the rate of formation of condensation product due to disturbance by the bulky $t$-butyl group, especially in the second amination process, resulting in different pathways in the $\mathrm{C}_{3}$ formation. Then, in the $t$-butylamine system the $\mathrm{C}_{3}$ product may possibly be formed according to the scheme
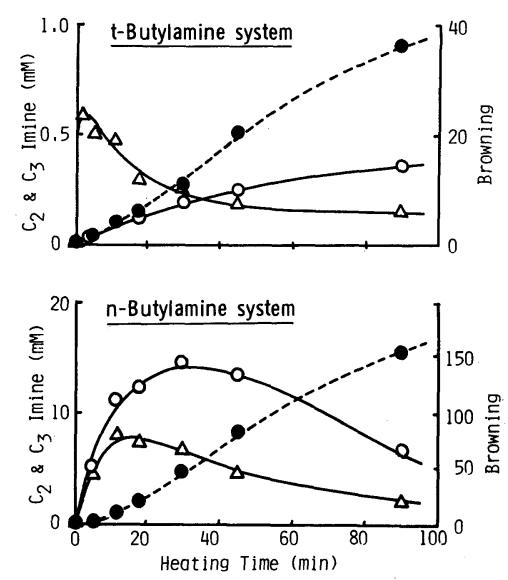

FIG. 7. Formation of $\mathrm{C}_{2}$ and $\mathrm{C}_{3}$ Imine and Browning during the Reaction of Glucose $(0.5 \mathrm{M})$ with $t$-Butylamine or $n$-Butylamine $(1 \mathrm{M})$ Heated at $80^{\circ} \mathrm{C}$.

$-\triangle-, \mathrm{C}_{2}$ imine; $-\mathrm{O}-\mathrm{C}_{3}$ imine; --- ---, browning.

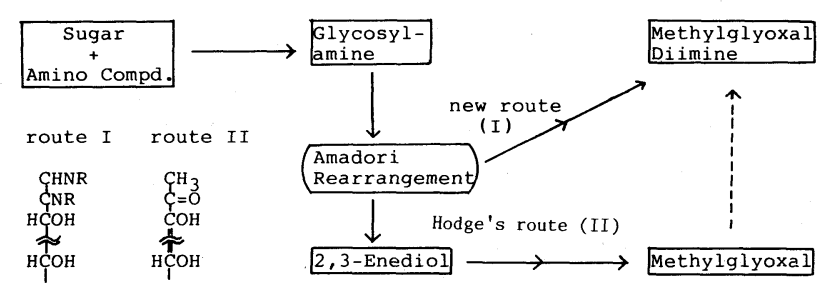

$\begin{array}{ll}\text { t-Butylamine System } & \text { route II } \\ \text { n-Butylamine System route I. and II }\end{array}$

FIG. 8. Proposed Pathway for $\mathrm{C}_{3}$ Imine Formation. 
proposed by Hodge, which is followed by deoxy-osone formation from the Amadori product at the middle stage of the aminocarbonyl reaction. On the other hand, the $\mathrm{C}_{3}$ formation in the systems of $n$-alkylamines may occur mainly via a newly proposed mechanism from the doubly aminated Amadori product. Two possible pathways of the $\mathrm{C}_{3}$. formation arising from these considerations are presented in Fig. 8. Further studies on the mechanism of this new pathway are underway.

\section{EXPERIMENTAL}

1. Materials and methods. Authentic specimens of glucosyl- $n$-butylamine and 1-deoxy-1-n-butylamino-fructose were prepared according to the literature. ${ }^{8)}$ Methylglyoxal bisphenylhydrazone was synthesized by the reaction of methylglyoxal with phenylhydrazine hydrochloride in aqueous sodium acetate, and its structure was confirmed by NMR. Other reagents were of guaranteed grade.

Analytical TLC was done on precoated Merck silica gel plates using the solvent system (EtOAc- $\mathrm{MeOH}-\mathrm{CHCl}_{3}$, $5: 2: 1)$. A solution of $2,4-\mathrm{DNP}(3 \%$ in the mixture of ethanol, water, and sulfuric acid $(70: 20: 15))$ was used for visualization and detection of the spots. TLC of phenylhydrazone was developed with benzene- $n$-hexane-EtOAc $(5: 2: 1)$.

NMR and mass spectra were recorded using JNMMH-100 and JMS-D-100 spectrometers, respectively. GC analysis was done on Hitachi GC $263-30$ on a $0.25 \mathrm{~mm}$ i.d. $\times 25 \mathrm{~m}$ fused silica capillary column coated with carbowax $20 \mathrm{M}$ at $170^{\circ} \mathrm{C}$. Preparative and analytical HPLC were done using a JEOL TWINCLE pump with Shodex RI SE-II.

Measurement of glucosylamine and the Amadori product formed from $n$-butylamine system was done by the previously reported method using HPLC. ${ }^{4)}$ The degree of browning was measured by the absorbancy at $420 \mathrm{~nm}$.

2. Isolation of phenylhydrazone from the reaction mixture. D-Glucose and $n$-butylamine $(0.1 \mathrm{~mol}$ each) in $100 \mathrm{ml}$ of distilled water were heated for $8 \mathrm{~min}$ in a boiling water bath and extracted with ether $(50 \mathrm{ml})$. The extract was washed with water three times $(50 \mathrm{ml}$ each). An aqueous solution $(16 \mathrm{ml})$ of phenylhydrazine hydrochloride $(1.5 \mathrm{~g})$ and sodium acetate $(2.4 \mathrm{~g})$ was added to the concentrated extract, and immediately extracted with ethyl acetate twice $(20 \mathrm{ml}$ each). The collected extract was washed with water two times $(20 \mathrm{ml}$ each) and then concentrated. Next, preparative TLC was done by using a silica gel plate (Wako Gel B, $0.5 \mathrm{~mm}$ thick, $20 \times 20 \mathrm{~cm}, 8$ sheets) with the same solvent as that of TLC for analysis. The topmost band among several yellow ones on TLC was scraped off and extracted with ethyl acetate. The dried matter
$(66.6 \mathrm{mg})$ gave yellow plates on crystallization with ethanol. The ethyl acetate extract was chromatographed by HPLC using a Lichrosorb SI60 column $(150 \times 8$ i.d. $\mathrm{cm})$, eluted with hexane-EtOAc $(90: 10)$ as solvent $(1.5 \mathrm{ml} /$ $\mathrm{min}$ ) and the fraction with retention time $11.3 \mathrm{~min}$ was collected.

3. Preparation of methylglyoxal dicyclohexylimine. A mixture prepared by the additon of methylglyoxal (10 $\mathrm{mmol})$ to an aqueous solution of cyclohexylamine $(22$ $\mathrm{mmol}$ ) in $10 \mathrm{ml}$ of water was immediately extracted with ether $(20 \mathrm{ml})$, and the extract was washed with water $(20 \mathrm{ml})$ four times, dried, and concentrated to $5 \mathrm{ml}$. After addition of a small portion of water, it was stored in a freezer overnight to yield a brown precipitate, which was crystallized from ethanol; yield $0.312 \mathrm{~g}$, pale brown and fine. MS $m / z$ (relative intensity); $234\left(\mathrm{M}^{+}, 10\right), 191$ $\left(\mathrm{C}_{12} \mathrm{H}_{19} \mathrm{~N}_{2+}, 66\right), 109$ (56), 96 (83), $83\left(\mathrm{C}_{6} \mathrm{H}_{11+}, 100\right), 52$ (91) and 40 (70). NMR, see Fig. 3.

4. Preparation of the ether extract of the reaction mixture of glucose and amine. The ether extract of a reaction mixture of glucose $(3.6 \mathrm{~g})$ and cyclohexylamine $(2.0 \mathrm{~g})$ in water $(20 \mathrm{ml})$, heated for $4 \mathrm{~min}$ in boiling water bath, was washed with water $(10 \mathrm{ml}) 5$ times and dried, and then made solvent-free $(0.18 \mathrm{~g})$.

An extract of an $n$-butylamine system was prepared under the conditions described previously. ${ }^{4}$

\section{Quantification of glyoxal and methylglyoxal dialkyl-} imine.

a) NMR method. A reaction mixture of glucose and $n$ butylamine was extracted with ethyl acetate. After washing with water, it was concentrated and dried, and then dissolved in $\mathrm{CDCl}_{3}$ containing TMS and 1,2-dichloroethane as standards. The amounts of $\mathrm{C}_{3}$ and $\mathrm{C}_{2}$ dialkylimine were calculated from the NMR signal intensities at 7.8 and $7.9 \mathrm{ppm}$, respectively, compared to that at $6.0 \mathrm{ppm}$ of the standard (1,2-dichloroethane).

b) o-Penylenediamine method. The GC measurement of quinoxaline derivatives was made by a modification of the method of Moree-Testa and Saint-Jalm. A testing mixture $(2 \mathrm{ml})$ was added to an aqueous solution of $o$-phenylenediamine $(1 \%, 10 \mathrm{ml})$, and mixed well with a magnetic stirrer for $30 \mathrm{~min}$. The dichloromethane $(2 \mathrm{ml})$ extract of the mixture, containing naphthalene as a standard, was analyzed by a Hitachi 263-30 GC with a capillary column (Carbowax $20 \mathrm{M}, 0.25 \mathrm{~mm} \times 25 \mathrm{~m}$ ).

Acknowledgments. We thank Messers K. Sekiya, S. Hayamizu, and Miss E. Sakamoto for their excellent technical assistance, and Dr. N. Ramarathnam for his helpful advice. This work was supported in part by a Grant-in-Aid for Scientific Research from the Ministry of Education, Science and Culture of Japan. 


\section{REFERENCES}

1) M. Namiki and T. Hayashi, J. Agric. Food Chem., 23, 487 (1974).

2) T. Hayashi, Y. Ohta and M. Namiki, J. Agric. Food Chem., 25, 1282 (1977).

3) T. Hayashi and M. Namiki, Agric. Biol. Chem., 44, 2575 (1980).

4) T. Hayashi, S. Mase and M. Namiki, Agric. Biol. Chem., 49, 3131 (1985).

5) T. Hayashi and M. Namiki, Agric. Biol. Chem., 45,
933. (1981).

6) J. E. Hodge, "Chemistry and Physiology of Flavors," ed. by Schulz, Day and Libbey, Avi Publishing Co., Westport, U.S.A., 1967, p. 465.

7) H. Budzikiewicz, C. Djerassi and D. H. Williams, "Mass Spectrometry of Organic Compounds," Holden-Day Inc., London, 1967, p. 308.

8) J. E. Hodge and B. E. Fisher, Method in Carbohydrate Chemistry, 2, 99 (1963).

9) P. Moree-Testa and Y. Saint-Jalm, J. Chromatogr., 217, 197 (1981). 